\title{
A Cross-Layer Control Based on Fuzzy Automata for Ad-Hoc WLAN QoS
}

\author{
Chao-Lieh Chen, Hsin-Long Yang, and Yao-De Huang \\ Department of Electronic Engineering \\ Kun-Shan University \\ Yung-Kang, Tainan County, TAIWAN \\ frederic@ieee.org
}

\begin{abstract}
This paper proposes a new interface model for crosslayer designs using generalized fuzzy automata (GFA) and fuzzy control. When using the proposed model, an upper layer is reduced to an adaptive and cognitive controller and the lower layers act as hierarchical plants. In this paper, we utilize the model in wireless LAN Quality of Service (QoS). The fuzzy controller allocates bandwidths for different traffics, detects saturation of performances, and criticizes satisfaction of traffic specifications (TSPECs). The distributed fuzzy controller adaptively tunes its fuzzy sets according to both the TSPECs and the network states. According to the realization theory and the experiments, the adaptive QoS controller fulfills TSPECs no matter what dynamics of the TSPECs and the network environment are.
\end{abstract}

Keywords: wireless ad-hoc networks, cross-layer design, fuzzy control, generalized fuzzy automata, IEEE 802.11e.

\section{Introduction}

The uncertain dynamics such as mobility, link capacity, and many other resources along with diverse QoS requirements in different applications make protocol designs significant challenges [1]. Scientists found that cross-layer designs are useful to tackle some of the challenges and will be essential for wireless networks. However, there are still many open challenges such as interface standardization, physical layer signal processing, co-existence problems, costeffectiveness assessments, and network states measurements [2]. This paper proposes a novel crosslayer interface model that provides certain levels of solutions to these challenges. As in Fig. 1, each of the lower layers interprets a control vector from upper layers as part of the dynamic command of its internal control system. Thus, the controller in a lower layer is transparent to an upper layer. The control system performances and costs can be analyzed in each logical layer by fuzzy feedback control theories.

This work is part of the project numbered NSC 94-2213-E-168-008- supported by the National

Science Council in TAIWAN
Different from the abstractions investigated in [2], our cross-layer proposal uses fuzzy automata and control to deal with the challenges respectively as follows. First, the proposed fuzzy control hierarchy does not violate the classical layered architecture but does promote efficiency and standardize the interactions between layers. Second, the interface model does not change the physical layer and the signal processing is as was in the classical OSI model. Third, since the layering abstraction is not completely destroyed, logical links between the same layer of different nodes are maintained. Therefore, the traditional and other cross-layer designs are able to communicate the proposed design. Moreover, costs and performances are easily assessed using control theories. We can thus perform cross-layer optimization using optimal fuzzy control. Finally, fuzzy automata theory is very useful in modeling and operating uncertain concepts. Uncertain states in the control system hierarchy are easily handled.

The utilization of the cross-layer model in WLAN QoS is as follows. The media access control (MAC) layer is based on the IEEE 802.11e standard [8], which receives dynamic TSPECs as commands from upper layer. In mobile ad-hoc networks, the TSPECs dynamically change due to flow control, traffic reshaping, or the mobile nodes recasting new roles in applications of scenarios. Moreover, a QoS algorithm requires the capabilities of determining resource availability on neighboring links and resource reservation functions at nodes [3]. Measurements at lower layers assure these capabilities. Therefore, the QoS work does not merely rely on a single layer but rather on all layers. How to accomplish the QoS is a typical example utilizing cross-layer designs and nodes' cooperation.

In the IEEE 802.11e MAC layer QoS standard [8], the Enhanced Distributed Channel Access Function (EDCAF) and the Hybrid Coordination Function (HCF) are alternatives to access the wireless channel. In this paper, we choose EDCAF as the plant in the fuzzy control system. Prioritized QoS is done by assigning of the parameters different values in different traffic categories. The parameters are the contention window $(\mathrm{CW})$, persistence factor $(\mathrm{PF})$, and arbitration inter- 
frame space (AIFS). The EDCAF function provides coordination for QoS enhancement. However, we suffer the following problems:

P1. Uncertainties and dynamics of network states: The measurement of network states is very difficult since there are too many affecting factors, which are unknown and dynamic.

P2. Dynamic TSPECs: Dynamic network state results in requirement of dynamic TSPEC. In the IEEE 802.11e standard, there is no definition of ways to realize dynamic TSPEC. In the MAC layer, we need to accurately fulfill or criticize both static and dynamic TSPECs requested by upper layers. Therefore, a cross-layer interface is required.

In this paper, we propose the cross-layer fuzzy control architecture to solve these problems. Many other recent studies on the QoS extension of wireless LAN can be found in [9-14]. These studies can be classified into two major categories. The first category papers focus on performance evaluation and analysis [9-10], where sophisticated stochastic models are used for dealing with the nonlinearities and uncertainty and the other is about control and enhancement of the IEEE 802.11e standard [11-14]. In the second category of studies, article [12] made the modification to acknowledgement (ACK) scheme of the original standard while some incorporated applications are proposed in [11-13]. In [13], we have developed the QoS control for legacy IEEE 802.11 to meet the node level dynamic TSPEC. In this paper, to support QoS required in different layers, we extend the controller into hierarchical and vectored fashion and prove its feasibility according to the realization theory. Instead of stochastic computing, we develop the soft computing model for the IEEE 802.11e EDCAF fuzzy control by generalized fuzzy automata theory and prove that the controller can be very simple, effective, and easy to realize. The remainder of this paper is organized as follows. Section II describes how the whole fuzzy controller is constructed based on GFA theory. In Section III, we give MPEG4 streaming simulations. In Section IV, we give conclusions and discussions.

\section{Realization of the Fuzzy Controller}

In this paper, we do not apply analytical procedure to find out exact probability model of IEEE $802.11 \mathrm{e}$ EDCAF functions in a cell. Instead, we apply fuzzy control to take care of uncertainties and non-linearity in wireless networks where states change quickly. According to the standard [8], a frame transmission in a channel will require the following time duration.

$d=t_{\text {defer }}+t_{R T S}+t_{C T S}+t_{\text {frame }}+t_{A C K}+3 t_{\text {SIFS }}+3 \tau_{p}$, where $t_{R T S}+t_{C T S}+t_{A C K}$ can be regarded as constant, $t_{\text {defer }}$ is the deferred time according to the specification of DCF, $t_{\text {frame }}$ is concerned with length of the frame, and $\tau_{p}$ is the maximum possible propagation delay. At MAC layer, the value of $t_{\text {frame }}$ is based on frame length and the data rate. To compatible with the standard, $t_{R T S}+t_{C T S}+t_{A C K}+3 t_{S I F S}$ is a constant. Therefore, the only adaptable parameter is $t_{\text {defer }}$, which is obtained by the back-off procedure defined in the standard. If the defer time of a traffic in a mobile node is effectively controlled, any dynamic TSPEC can be realized.

\subsection{Automaton for the Adaptive Controller}

The control system architecture is shown as Fig. 2. Suppose there are $n$ nodes each of which at most has $m$ traffic categories. Every node $i$ has basic rate $B_{i}$ and the total network capacity is $B$. The distributed controller has two sets of inputs - TSPEC and the observed system state. One element of the TSPEC input set is the goal delay matrix $\left[D_{g i}\right]$ where each $D_{g i}$ is a row vector of $m$ goal delays $d_{i j}$ 's for traffic categories $j$ 's of node $i$. Each goal delay $d_{i j}$ is related with the packet size $l_{i j}$ and the desired bandwidth $b_{i j}$. Thus we have

$$
\begin{aligned}
& B=\sum_{i=0}^{n-1} B_{i}=\sum_{i=0}^{n-1} \sum_{j=0}^{m-1} b_{i j} \\
& d_{g i j}=\frac{l_{i j}}{b_{i j}}, j>0 ; d_{g i 0}=\frac{l_{i 0}}{B_{i}-\sum_{j=1}^{m-1} b_{i j}}
\end{aligned}
$$

The traffic category $(j=1)$ with lowest priority uses the remaining bandwidth for transmission. The TSPEC includes the tolerable delay variation matrix $\left[E_{i}\right]$ where each $E_{i}$ is a row vector of $m$ desired tolerable delay variations $\varepsilon_{i j}$ 's for traffic categories $j$ 's of node $i$. In the network, the delay vector $D_{g i}$ and the tolerable delay variation $E_{i}$ are dependent.

To model the control system with the generalized fuzzy automata (GFA) [4, 14], we regard the level-2 fuzzification $Q$ of the observed network states $\xi_{m n}$ 's as the state universe of the automaton while the adaptive distributed controller becomes the output function that gives the actions $U_{m n}=\left[C W \min _{i j}\right]_{m \times n}$. Let $F$ : TSPEC $\rightarrow$ $\left[D_{g i}\right]$ be the transformation as equation (2), $\xi_{0}=\xi_{m n}(0)$ be the initial state, $\Sigma$ be the range of $C W$ min $_{i j}$ parameter, and $T$ be the set of fuzzy time durations $\left\{d T_{r} \mid r \in \mathbb{N}\right\}$ that the state transition $\delta=\left\{\delta\left(u_{r}(t), \xi_{r}(t)\right.\right.$, $\left.\left.d T_{r}\right) \mid r \in \mathbb{N}\right\}$ requires for changing from one fuzzy state to another. Then, we have $m \times n$ distributed GFA's $\boldsymbol{M}_{\boldsymbol{m} \boldsymbol{n}}=\left\{\boldsymbol{M}_{i j}\left(\delta, \Sigma, Q, \xi_{0}, F, T\right) \mid 0<i \leq n, 0<j \leq n\right.$ and $i, j$ $\in \mathbb{N}\}$ realizing the control system if the transition rule base $\delta$ can be found. Unfortunately, since the whole system is dynamic and uncertain, it is impossible to find out exact $\delta$ via classical system identification 
approaches. Moreover, even some very accurate system models of the EDCAF function can be found by delicacy analyses [9-10], as [11] mentioned it is still very difficult to have the sophisticated probability model of the whole system behavior without specific assumptions. The system behavior can be only be described by observations. Defining $\xi_{i j}$ as the error between the desired and measured MAC access delays, by using the observer in Fig. 2, we only know that by giving $C W \min _{i j}=\operatorname{Small}(S), \operatorname{Medium}(M)$, or Large $(L)$ the observed maximum and minimum state (delay) changing rates $r_{i j}$ and $l_{i j}$ in either directions of state axis can be found as follows.

$$
\begin{aligned}
& C W \min _{i j}=\text { Small, }-r_{i j}<d \xi_{i j} / d t<-l_{i j}<0 \\
& C W \min _{i j}=\text { Medium }, d \xi_{i j} / d t \approx 0 \\
& C W \min _{i j}=\text { Large, } 0<l_{i j}<d \xi_{i j} / d t<r_{i j}
\end{aligned}
$$

This is a simple input-state observation and therefore the observation is achievable [5]. We obtain the state and its changing rates $r_{i j}$ and $l_{i j}$ by the observer in Fig. 2 that performs simple histogram analysis - averaging packet delays during a time window. Thus, the state transition $\delta$ is an input-state homomorphism of the above vague information $[5,14]$. To attain the goal, we design the feedback controller for the automaton $\boldsymbol{M}_{i j}$ is as Fig. 3. The fuzzy sets $P$ and $N$ represent fuzzy concepts "Positive" and "Negative" error values respectively while $P$ ' and $N$ ' represent fuzzy concepts "Positive" and "Negative" error changing rates respectively. The domains of base variables error (denoted $e$ ), $\Delta$ error $/ \Delta t$ (denoted $e^{\prime}$ ), and $C W \min _{i j}$ are $\left[Q_{1}(t), Q_{2}(t)\right], \quad\left[d Q_{1}(t), d Q_{2}(t)\right]$, and $\left[\Sigma_{1}(t), \quad \Sigma_{2}(t)\right]$ respectively. The conclusion parts of the four rules use singletons representing Small (S), Medium (M), or Large (L) quantity of $C W \min _{i j}$. In Fig. 3, the value of the base variable error is the error between the desired delay and the system output. It is obtained by subtracting the desired delay $d_{g i j}$ from the observed control system output $\boldsymbol{O}\left(\xi_{i j}(t)\right)$. The membership functions of the fuzzy sets of the controller are timevariant and they are on-line tuned according to network status.

\subsection{Controller Adaptation and Cross-Layer Signaling}

The membership functions of the controller rule base are specified in terms of the TSPEC including goal delay, tolerable delay variation while the upper layers determine the TSPEC according to the measured MAC access delay and loss rate. The membership functions are as follows, where the symbol Өrepresents the bounded difference operations.

$$
\mu_{S}\left(C W \min _{i j}, t\right)=\left\{\begin{array}{cc}
1, & C W \min _{i j}=d_{g i j}(t)-\varepsilon_{i j}(t) \\
0, & \text { otherwise }
\end{array}\right.
$$

$$
\begin{aligned}
& \mu_{M}\left(C W \min _{i j}, t\right)=\left\{\begin{array}{lc}
1, & C W \min _{i j}=d_{g i j}(t) \\
0, & \text { otherwise }
\end{array}\right. \\
& \mu_{L}\left(C W \min _{i j}, t\right)=\left\{\begin{array}{lc}
1, & C W \min _{i j}=d_{g i j}(t)+\varepsilon_{i j}(t) \\
0, & \text { otherwise }
\end{array}\right. \\
& \mu_{P \cup N}(e, t)=1 \ominus \frac{C W \max _{i j}(t)-|e|}{C \max _{i j}(t)} \\
& \mu_{P^{\prime} \cup N^{\prime}}\left(e^{\prime}, t\right)=1 \ominus \frac{C W \max _{i j}(t)-\left|e^{\prime}\right|}{2 C \max _{i j}(t)}
\end{aligned}
$$

For the feedback signaling to upper layers, the measured loss rate and delay are used to estimate the noise and background traffic and then the sending rate, compression factors, and packet size are adapted. Furthermore, they are also useful in the modulation scheme selection of the physical layer. When measuring the delay, we perform the weighted moving average [13] of all frame delays in a sliding window. The losses of packets are found if the number of retries to access the channel exceeds the retry limits RetryLimit $_{i j}$ as defined in the standard.

\section{MPEG4 Streaming Simulations}

At time 0 , the MPEG4 streaming with packet size 1024 bytes is from node 0 to node 2 where there is also accompanied a background traffic $300 \mathrm{kbps}$ at packet size $1 \mathrm{k}$ bytes. There are four pairs of nodes and each pair is also generating background $300 \mathrm{kbps}$ at packet size $1 \mathrm{k}$ bytes. The AIFS are all 50 slots and each slot is $20 \mu \mathrm{s}$. The other parameters are as Table. 1. Fig. 4 shows the throughput where the fairness among the nodes is preserved. From Fig. 5, 6, and Table 2, we can compare the video results via both human visual feeling and the PSNR curves. We use the NS2 simulator for the network and protocol stacks environment. We use MATLAB and FPGA hardware for the fuzzy controller. These two tools pass data via sockets in the personal computer.

\section{Conclusions}

This paper proposes a new cross layer model which utilizes fuzzy control and generalized fuzzy automata theory to successfully tackle the uncertainty and dynamic problems in ad-hoc wireless networks. The QoS control based on the proposed approaches features better video streaming quality and at the same time preserves the fairness.

\section{References}

[1] S. L. Kota, E. Hossain, R. Fantacci, and A. Karmouch, "Crosslayer protocol engineering for wireless mobile networks-I," IEEE Communications Magazine, vol. 43, no. 12, Dec. 2005, pp. $110-111$. 
[2] V. Srivastava, M. Motani, "Cross-layer design: a survey and the road ahead," IEEE Communications Magazine, vol. 43, no. 12, Dec. 2005, pp. $112-119$.

[3] B. Zhang and H. T. Mouftah, "QoS Routing for Wireless Ad Hoc Networks:Problems, Algorithms, and Protocols," IEEE Communications Magazine, vol. 43, no. 10, Oct. 2005, pp. 110-117.

[4] Chao-Lieh Chen, "Programmable fuzzy logic device for sequential fuzzy logic synthesis", The 10th IEEE International Conference on Fuzzy Systems, Vol. 1, Dec. 2001, pp.107 - 110

[5] S. S. L. Chang and L. A. Zadeh, "On fuzzy mapping and control", IEEE Tran. Syst., Man, and Cybernet., Vol. 2, No. 1, Jan. 1972, pp. 30-34.

[6] Yau-Hwang Kuo; Chao-Lieh Chen, "Generic LR fuzzy cells for fuzzy hardware synthesis," IEEE Transactions on Fuzzy Systems, Vol. 6, No. 2, May 1998, pp. $266-285$.

[7] Yau-Hwang Kuo, Chang-Shing Lee, Chao-Lieh Chen, "Highstability AWFM filter for signal restoration and its hardware design," Fuzzy Sets and Systems, Vol. 114, No. 2, Sep, 2000, pp. 185-202.

[8] Wireless LAN Medium Access Control (MAC) and Physical Layer (PHY) Specifications: Amendment 8: Medium Access Control (MAC) Quality of Service ( $Q \mathrm{OS})$ Enhancement, IEEE Standard 802.11e, Sept. 2005.

[9] Zhu Hua and I. Chlamtac, "Performance analysis for IEEE 802.11e EDCF service differentiation," IEEE Transactions on Wireless Communications, vol. 4, no. 4, July 2005 pp. 1779 1788.

[10] Yang Xiao, "Performance analysis of priority schemes for IEEE 802.11 and IEEE 802.11e wireless LANs," IEEE Transactions on Wireless Communications, vol. 4, no. 4, July 2005 , pp. $1506-1515$.

[11] A. Banchs and A. Azcorra, et. al. "Applications and challenges of the 802.11e EDCA mechanism: an experimental study," IEEE Network, vol. 19, no. 4, July-Aug. 2005, pp. 52 - 58.

[12] L. Vollero, A. Banchs, and G. Iannello, "ACKS: a technique to reduce the impact of legacy stations in 802.11e EDCA WLANs," IEEE Communications Letters, vol. 9, no. 4, Apr. 2005 pp. $346-348$.

[13] Chao-Lieh Chen and Po-Chien Hsiao, "Supporting QoS in wireless MAC by fuzzy control," Proceedings of IEEE Wireless Communications and Networking Conference WCNC'05, New Orleans, March 2005, Vol. 2, pp. 1242 - 1247.

[14] Chao-Lieh Chen, "Generalized fuzzy automata for Fuzzy feedback control with words," Proceedings of 2004 International Computer Symposium ICS2004, Taiwan, Dec 1517, 2004, pp. 895-900.

Table 1. Parameters for EDCAF and proposed fuzzy control.

\begin{tabular}{|l|c|c|c|c|c|c|}
\hline & AIFS & CWmin & CWmax & $\begin{array}{c}\text { Retry } \\
\text { limit }\end{array}$ & $\begin{array}{c}\text { Pkt. Size } \\
\text { Bytes }\end{array}$ & $\begin{array}{c}\text { Data rate } \\
\text { kbps }\end{array}$ \\
\hline MPEG4(TC2) & 50 & \multicolumn{2}{|c|}{ Fuzzy Control } & 8 & Max1028 & Mean400 \\
\hline Bkground(TC1) & 50 & 31 & 1023 & 4 & 1000 & 300 \\
\hline
\end{tabular}

Table 2. Losses and PSNR comparisons.

\begin{tabular}{|l|l|l|l|}
\hline & Skipped frames & Lost packets & Video PSNR \\
\hline EDCA & 190 & 397 & $30.54 \mathrm{~dB}$ \\
\hline Proposed & 42 & 93 & $36.16 \mathrm{~dB}$ \\
\hline
\end{tabular}

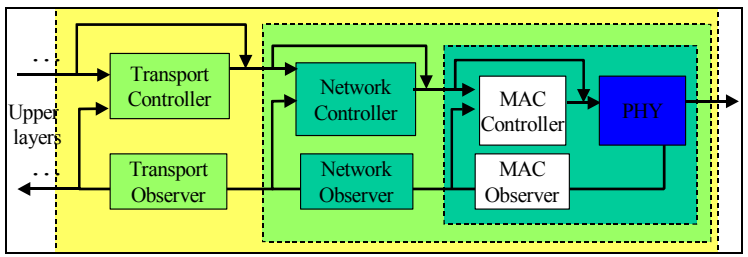

Fig. 1: Hierarchical cross-layer interface model.

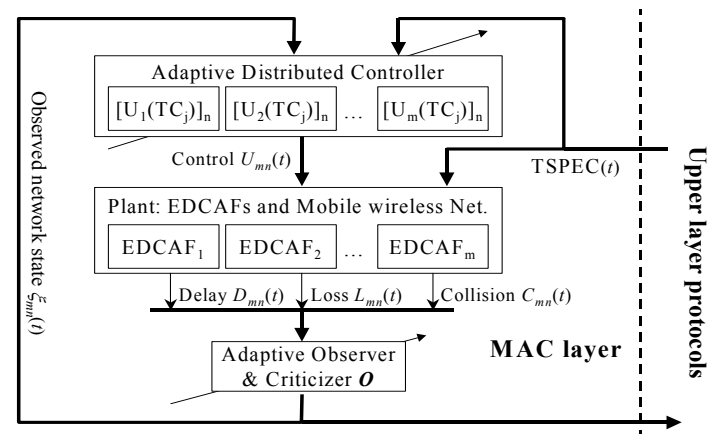

Fig. 2: The architecture of the 802.11 e control system.

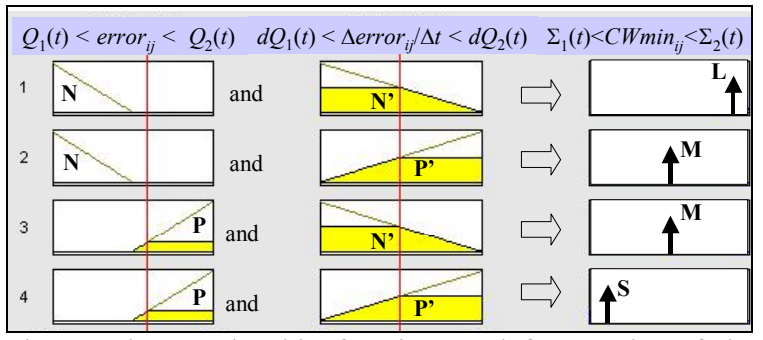

Fig. 3: The membership functions and fuzzy rules of the fuzzy controller.

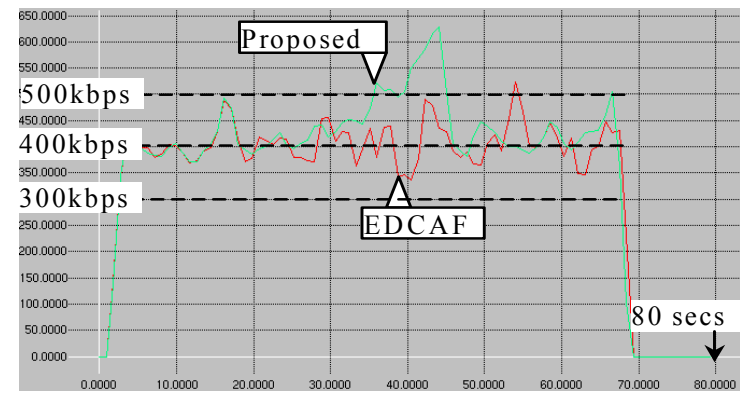

Fig. 4: Throughput comparison.

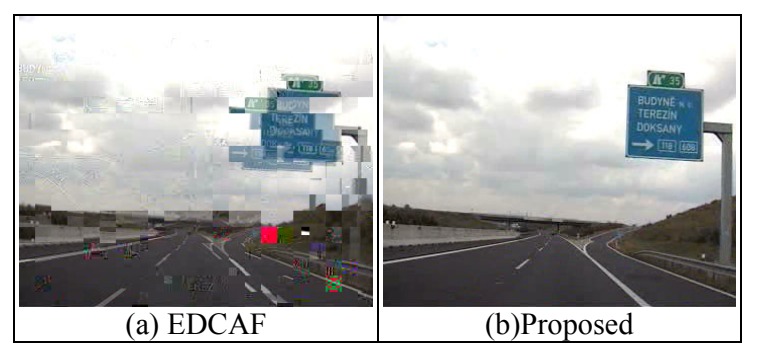

Fig. 5. Video captured from the NS2 simulator.

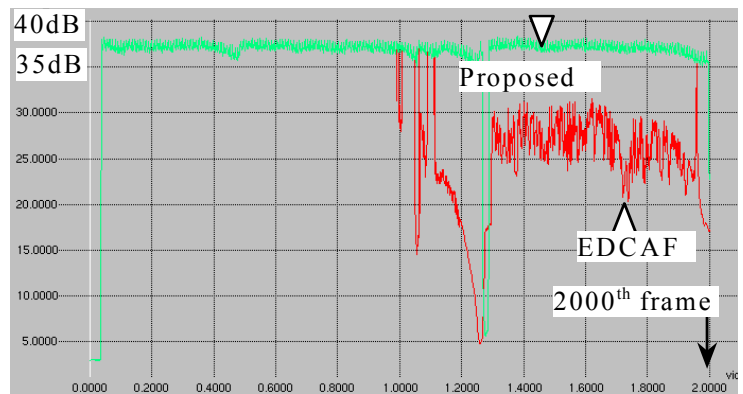

Fig. 6: Comparison of the PSNR performance. 